\title{
Curves Disjoint from a Nef Divisor
}

\author{
John Lesieutre \& John Christian OtTem
}

\begin{abstract}
On a projective surface it is well known that the set of curves orthogonal to a nef line bundle is either finite or uncountable. We show that this dichotomy fails in higher dimension by constructing an effective, nef line bundle on a threefold that is trivial on countably infinitely many curves. This answers a question of Totaro. As a pleasant corollary, we exhibit a quasi-projective variety with only a countably infinite set of complete, positive-dimensional subvarieties.
\end{abstract}

\section{Introduction}

If $L$ is a nef line bundle on a smooth complex projective surface, then the set of curves $C$ such that $L \cdot C=0$ is either finite or uncountable (when some such $C$ moves in a positive-dimensional family). This follows essentially from the Hodge index theorem (see Section 1.1). In [7], Totaro asked whether this remains true in higher dimensions:

Question. Is there a nef line bundle $L$ on a normal complex projective variety $X$ such that the set of curves $C$ with $L \cdot C=0$ is countably infinite?

In this note we construct examples of such $L$ in any dimension greater than two, which are in fact effective and movable divisors. Perhaps the surprising thing is not that such examples exist, but that they turn out to be so accessible: our example is the blow-up of $\mathbb{P}^{3}$ at eight very general points, and $L$ is the anticanonical divisor. Our main result is the following:

THEOREM 1. There exists a smooth projective rational threefold $X$ with nef anticanonical divisor such that the set of curves $C$ with $-K_{X} \cdot C=0$ is countably infinite and Zariski dense.

In particular, since $-K_{X}$ is effective in the example, the complement of the zero set of a global section gives an example of the following:

COROLlary 2. There exists a quasi-projective variety with only a countably infinite set of complete, positive-dimensional subvarieties.

We show in Corollary 7 that this is impossible in dimension less than 3. Furthermore, we show that the question has an affirmative answer even if the line bundle is required to be big and nef, which is impossible in dimension less than four (cf. Remark 13).

Received February 16, 2015. Revision received July 28, 2015. 
COROLlaRY 3. There exists a smooth projective fourfold $Y$ and a big and nef line bundle $M$ on $Y$ such that the set of curves $C$ with $M \cdot C=0$ is countably infinite.

Note that when the line bundle $L$ is semiample, these sorts of pathologies do not occur. In that case, some multiple of $L$ defines a morphism to projective space $X \rightarrow \mathbb{P}^{N}$ that contracts exactly the curves orthogonal to $L$, so this locus is Zariski closed. In particular, if $X$ is a Mori dream space, then every nef line bundle is semiample and so is zero on an either finite or uncountable set of curves. This includes all examples in which $X$ is the blow-up of $\mathbb{P}^{3}$ at $r \leq 7$ points.

\subsection{The Surface Case}

Here we prove a slight generalization of Totaro's result [7] for the dimension 2 case. This result will be needed in the proof of Theorem 1.

Proposition 4. Suppose that $S$ is a complex projective surface and let $L$ be a line bundle on $S$ (not necessarily nef). Then the set of irreducible curves $C$ with $L \cdot C=0$ is either finite or uncountable. Moreover, when $L$ is nef, the set of curves $C \notin \mathbb{R}_{>0} L$ with $L \cdot C=0$ is finite, and all these curves have negative selfintersection.

We note that the set defined is uncountable if and only if there exists an algebraic family of curves $C_{t}$ such that $L \cdot C_{t}=0$ for all $t$. This follows since the Hilbert scheme has only countably many components.

Proof of Proposition 4. It suffices to prove the result for $S$ smooth. Indeed, if $\pi$ : $S^{\prime} \rightarrow S$ is a desingularization of $S$, and $L^{\prime}=\pi^{*} L$, then the curves with $L^{\prime} \cdot C=0$ are the (finitely many) curves contracted by $\pi$, together with the transforms of the curves with $L \cdot C=0$.

Let $\mathscr{C}$ denote the set of irreducible curves $C$ on $X$ such that $L \cdot C=0$. We assume for a contradiction that $\mathscr{C}$ is countably infinite.

Suppose first that $L$ is a nef line bundle. If $L^{2}>0$, then $L$ is big, and there are at most $\rho-1$ curves with $L \cdot C=0$, and they all satisfy $C^{2}<0$ by the Hodge index theorem. If $L^{2}=0$, then there can be at most $2(\rho-2)$ curves $C \in \mathscr{C}$ with $C \notin$ $\mathbb{R}_{>0} L$, and they all satisfy $C^{2}<0$. Indeed, changing the sign on the intersection form defines an inner product on $V=L^{\perp} / \mathbb{R} L \simeq \mathbb{R}^{\rho-2}$, and the claim follows by Lemma 6 below. Furthermore, Totaro showed that given three pairwise disjoint connected effective divisors $D_{1}, D_{2}, D_{3}$ with proportional divisor classes, there is a map $f: S \rightarrow B$ to a smooth curve such that the $D_{i}$ are all rational multiples of fibers of $f[8, \mathrm{Thm} .2 .1]$. Applying this to our situation, we see that a stronger statement holds: Given any set of $r>2 \rho$ distinct curves $C_{1}, \ldots, C_{r}$ so that $L$. $C_{i}=0$, one of the $C_{i}$ has a rational multiple that moves in a family. In particular, if $|\mathscr{C}|>2 \rho$, then $\mathscr{C}$ is uncountable.

Suppose now that $L$ is an arbitrary line bundle, not necessarily nef. Pick $\rho$ distinct curves $C_{1}, \ldots, C_{\rho}$ such that $L \cdot C_{i}=0$. Then in $N^{1}(S)$, we must have a 
relation between them of the form

$$
m_{1} C_{1}+\cdots+m_{s} C_{s} \equiv m_{s+1} C_{s+1}+\cdots+m_{\rho} C_{\rho},
$$

where $m_{i} \geq 0$ are integers. Let $D_{1}$ (resp. $D_{2}$ ) denote the effective divisor on the left-hand side (resp. right-hand side) of this equation. Given any irreducible curve $C$, at least one of $D_{1}$ or $D_{2}$ does not contain $C$ in its support, and so either $D_{1} \cdot C \geq 0$ or $D_{2} \cdot C \geq 0$. But $D_{1}$ and $D_{2}$ are numerically equivalent, and we thus conclude that $D_{1}$ (and hence $D_{2}$ ) is nef.

We claim next that there exists a curve $C \in \mathscr{C}$ such that $D_{1} \cdot C>0$. Indeed, if $|\mathscr{C}|>2 \rho$ and every curve in $\mathscr{C}$ has $D_{1} \cdot C=0$, then some element of $\mathscr{C}$ moves in a family by the preceding arguments, applied to the nef class $D_{1}$. But then $\mathscr{C}$ is uncountable. Now, for $\epsilon>0$ small and rational, the $\mathbb{Q}$-divisor $M=D_{1}+\epsilon C \in \mathscr{C}$ is nef and big. Taking $n$ large and divisible, the linear system $|n M|$ has positive dimension, and the fixed components are supported on the curves $C_{1}, \ldots, C_{s}, C$ that all lie in $\mathscr{C}$. After subtracting the fixed components, we again obtain a positivedimensional family of curves $C_{t}$ such that $L \cdot C_{t}=0$. This contradiction completes the proof.

REMARK 5. The proof shows that the proposition also holds when $L$ is an $\mathbb{R}$ divisor.

Lemma 6. Suppose that $V=\mathbb{R}^{n}$ and $x_{1}, \ldots, x_{m}$ are distinct, nonzero vectors spanning $V$ such that $x_{i} \cdot x_{j} \leq 0$ for $i \neq j$. Then it must be that $m \leq 2 n$.

Proof. We show that in fact if $m \geq n+2$, there exist pairwise orthogonal linear subspaces $\ell_{1}, \ldots, \ell_{m-n}$ of $V$ such that each $\ell_{i}$ contains $\operatorname{dim} \ell_{i}+1 x_{i}$ s that $\operatorname{span} \ell_{i}$. The maximal $m$ is then achieved when each of the $\ell_{i}$ is one-dimensional, so that $m=2 n$.

We proceed by induction on $n$, the case $n=2$ being clear. For $n>2$, we may assume that each $x_{i}$ does not point in the opposite direction of $x_{n}$ (in which case $x_{j} \in x_{n}^{\perp}$ for each $j \notin\{i, n\}$, and we instead consider $\left.V=x_{n}^{\perp}\right)$. Write $x_{i}=y_{i}+\lambda_{i} x_{n}$, where $y_{i} \in x_{n}^{\perp}$ and $\lambda_{i} \leq 0$. We find that $y_{i} \cdot y_{j} \leq 0$ for $i \neq 0$, with strict inequality if both $\lambda_{i}, \lambda_{j} \neq 0$. By induction, we obtain orthogonal linear subspaces $\ell_{1}^{\prime}, \ldots, \ell_{m-n}^{\prime}$ of $x_{n}^{\perp}$ satisfying the conclusions of the lemma. Without loss of generality, we may assume that $\ell_{1}^{\prime}$ contains every $y_{i}$ such that $\lambda_{i}<0$. Then define $\ell_{1}=\ell_{1}^{\prime}+\mathbb{R} x_{n}$ and $\ell_{i}=\ell_{i}^{\prime}$ for $i>1$.

When $L=\mathcal{O}_{S}(D)$ is effective and the number of curves orthogonal to $L$ is finite, the previous proof shows that it is bounded by a number depending only on the Picard number of $S$. In fact, as remarked in [7], the bound 2( $\rho-2)$ for the number of negative curves in $L^{\perp}$ is even sharp; it can be attained, for example, on the blow-up of $\mathbb{P}^{1} \times \mathbb{P}^{1}$ at $\rho-2$ generic points, letting $L$ be the pullback of one of the fibers. Interestingly, the proof of Lemma 6 also shows that the value $2(\rho-2)$ is obtained in general if and only if we can partition the curves into $\rho-2$ sets of mutually orthogonal pairs of classes, as in the example. 
Corollary 7. Suppose that $U$ is a quasiprojective surface over $\mathbb{C}$. Then the set of complete curves in $U$ is either finite or uncountable.

Proof. Let $X$ be a compactification of $U$ with $\pi: Y \rightarrow X$ a resolution of $X$, and set $V=\pi^{-1}(U)$. If $C$ is a complete curve contained in $V$, then either $C$ is contracted by $\pi$, or $\pi(C)$ is a complete curve contained in $V$. Since only finitely many curves are contracted, it suffices to prove the claim for $V$. Consider the boundary $\Delta=Y-V$. The complete curves in $V$ are precisely the curves $C$ on $Y$ that are not components of $\Delta$ but satisfy $\Delta \cdot C=0$. By Proposition 4 the set of these curves is either finite or uncountable.

\section{The Rational Threefold}

Let $p_{1}, \ldots, p_{8}$ be eight very general points in $\mathbb{P}^{3}$. By this we mean that the points are chosen from the complement of a countable union of closed subvarieties in $\left(\mathbb{P}^{3}\right)^{8}$; exactly which subvarieties of the parameter space are to be avoided will be made precise later. Since the points are general, the linear system of quadric surfaces containing the points is a one-dimensional pencil. Let $Q_{0}, Q_{1}$ be two distinct smooth quadric surfaces in this pencil. The base-locus of the pencil, $B=$ $Q_{0} \cap Q_{1}$, is a smooth genus 1 curve, which is a bidegree $(2,2)$ divisor on the quadrics.

Define $\pi: X \rightarrow \mathbb{P}^{3}$ to be the blow-up of $\mathbb{P}^{3}$ at the points $p_{1}, \ldots, p_{8}$ and let $B^{\prime}$ be the strict transform of $B$ on $X$. The pencil of quadrics determines a rational map $f: X \rightarrow \mathbb{P}^{1}$ defined outside the curve $B^{\prime}$. The fibers of this map are blowups of quadric surfaces in the eight points $p_{1}, \ldots, p_{8}$.

Write $H=\pi^{*} \mathcal{O}_{\mathbb{P} 3}(1)$ and let $E_{1}, \ldots, E_{8}$ be the exceptional divisors of $\pi$. Similarly, let $h=H^{2}$ be the class of the transform of a general line in $\mathbb{P}^{3}$, and let $e_{1}, \ldots, e_{8}$ denote classes of lines in $E_{1}, \ldots, E_{8}$ (which are projective planes). We have $N^{1}(X)=\mathbb{Z} H \oplus \mathbb{Z} E_{1} \oplus \cdots \oplus \mathbb{Z} E_{8}$ and $N_{1}(X)=\mathbb{Z} h \oplus \mathbb{Z} e_{1} \oplus \cdots \oplus \mathbb{Z} e_{8}$, and we will call these generators the standard bases for $N^{1}(X)$ and $N_{1}(X)$.

Let $L=-K_{X}$ be the anticanonical divisor of $X$ : this is the nef divisor we are looking for. In terms of this basis, the canonical divisor is given by $-4 H+$ $2 E_{1}+\cdots+2 E_{8}$. Note that $L$ is nef since its base-locus is exactly the curve $B^{\prime}$ and $L \cdot B^{\prime}=-K_{X} \cdot B^{\prime}=\left(-K_{X}\right)^{3}=0$. Note also that the fibers of $f$ correspond to divisors in the linear system $\left|-\frac{1}{2} K_{X}\right|$.

There are many curves $C$ such that $L \cdot C=0$. For example, let $C$ be the strict transform of the line $l$ through the points $p_{1}$ and $p_{2}$. The normal bundle of $C$ is isomorphic to $\mathcal{O}(-1) \oplus \mathcal{O}(-1)$ and the class of $C$ is $h-e_{1}-e_{2}$. Furthermore, we have

$$
L \cdot C=-K_{X} \cdot C=\operatorname{deg} N_{C}+\operatorname{deg} K_{C}=-2+2=0 .
$$

Let $Q$ be the quadric surface in the pencil containing $l$ as one of its rulings. Then the strict transform $S$ of $Q$ is the blow-up of $Q$ in $p_{1}, \ldots, p_{8}$, and the curve $C$ is a $(-2)$-curve on $S$ (i.e., a smooth rational curve of self-intersection -2 ), with class $\pi^{*} \mathcal{O}(0,1)-E_{1}-E_{2}$ in $\operatorname{Pic}(S)$. 
The same thing happens if we take $C$ to be the strict transform of a twisted cubic curve in $\mathbb{P}^{3}$ through six of the points $p_{1}, \ldots, p_{6}$; the class of $C$ is $3 h-$ $e_{1}-\cdots-e_{6}$, and $L \cdot C=0$. There is a unique quadric surface $Q$ in the pencil containing the twisted cubic, and its strict transform $S$ contains $C$ as a $(-2)$ curve.

In fact, we will show below that there are countably infinitely many curves $C$ on $X$ such that $L \cdot C=0$ : these will be constructed as the strict transforms of the lines in $\mathbb{P}^{3}$ through a pair of points and under sequences of Cremona transformations on $\mathbb{P}^{3}$ based at quadruples of points. These are all rigid rational curves with normal bundle isomorphic to $\mathcal{O}(-1) \oplus \mathcal{O}(-1)$. Moreover, the set of these curves is Zariski dense in $X$.

LeMma 8. Let $S$ be a smooth rational surface with $-K_{S}$ nef and $K_{S}^{2}=0$. If $C$ is an irreducible curve such that $K_{S} \cdot C=0$, then either $C \in\left|-m K_{S}\right|$ for some integer $m \geq 1$, or $C$ is a smooth rational curve of self-intersection -2 .

Proof. The Hodge index theorem implies that $C^{2} \leq 0$. If $C^{2}<0$, then $C \cdot K_{S}=0$ implies $C^{2}=-2$ and $p_{a}(C)=0$ by the adjunction formula, and hence $C \simeq \mathbb{P}^{1}$. So suppose $C^{2}=0$. For any $C^{\prime} \in K_{S}^{\perp}$, either $C^{\prime} \cdot C=0$, or we have $\left(t C+C^{\prime}\right)^{2}>0$ for some $t>0$. In the latter case we again obtain a contradiction using the Hodge index theorem. Thus, we have that $C^{\perp}=K_{S}^{\perp}$, and hence $C=-m K_{S}$ for some $m \geq 1$ since $-K_{S}$ is not divisible in $\operatorname{Pic}(S)$ (since a rational surface with $K_{S}^{2}=0$ contains a $(-1)$-curve $C$, and $\left.K_{S} \cdot C=-1\right)$.

So far we have not used the fact that the points $p_{1}, \ldots, p_{8}$ are very general on $B$, meaning that they are chosen in the complement of a countable union of subvarieties of $B^{8}$. This is essential for the following result.

Lemma 9. Let $B \subset \mathbb{P}^{3}$ be a smooth quartic curve of genus 1 . Then for a very general $Q$ in the pencil, there are no relations in $\operatorname{Pic}(B)$ between line bundles on $Q$ and the points $p_{1}, \ldots, p_{8}$.

Proof. We first claim that the line bundle $\mathcal{O}_{Q}(1,-1)$ is nontorsion for some smooth quadric $Q$ in the pencil. Indeed, fix two points $p, q \in B$ such that $p-q \in \mathrm{Pic}^{0}(B)$ is nontorsion and the divisor $2 p+2 q$ embeds $E$ into a smooth quadric $Q \subset \mathbb{P}^{3}$. Then $\mathcal{O}_{Q}(1,-1)$ pulls back to $2 p-2 q$, which is nontorsion.

Now, let $M$ be a line bundle on $Q$. For each set of integers $a_{1}, \ldots, a_{8}$, not all zero, such that $M^{\prime}=\left.M\left(-a_{1} p_{1}-\cdots-a_{8} p_{8}\right)\right|_{B}$ has degree 0 on $B$, there is a proper Zariski closed subset of points $\left(p_{1}, \ldots, p_{8}\right) \in B^{8}$ such that the $M^{\prime}$ is effective on $B$ (that is, $\left.M^{\prime}\right|_{B}=\mathcal{O}_{B}$ ). If the points $p_{1}, \ldots, p_{8}$ are chosen outside the countable union of all these closed subsets running through all the choices $M, a_{1}, \ldots, a_{8}$, then no nontrivial line bundle on $Q$ restricts to the trivial bundle on $B$.

Now by semicontinuity the same statement remains true for a very general quadric in the pencil. 
We are now ready to prove the main result of this section.

Lemma 10. The set of curves $C \subset X$ for which $L \cdot C=0$ is at most countably infinite.

Proof. Suppose that $C$ is an irreducible curve with $L \cdot C=0$. If $C$ is not contained in the base locus of $\left|-\frac{1}{2} K_{X}\right|$, then $C$ meets some fiber $S \in\left|-\frac{1}{2} K_{X}\right|$ of the map $f: X \rightarrow \mathbb{P}^{1}$ at a point not contained in $B^{\prime}$. If $C$ is not contained in the fiber $S$, then $S \cdot C$ is positive, and so too is $L \cdot C$. Consequently, if $C$ is a curve with $L \cdot C=0$, then either $C$ is the unique curve $B^{\prime}$ in the base locus, or $C$ lies in some fiber $S$ of $f$. In the following, we will assume that $C \neq B^{\prime}$.

Assume first that $S$ is smooth. Then $S$ is the strict transform of a smooth quadric, that is, the blow-up of $\mathbb{P}^{1} \times \mathbb{P}^{1}$ along eight points. By Lemma $8, C$ is either linearly equivalent to a multiple of $-K_{S}=B^{\prime}$ or is a $(-2)$-curve on $S$. However, the normal bundle of $B^{\prime}$ in $S$ is

$$
\left.\mathcal{O}_{S}\left(B^{\prime}\right)\right|_{B^{\prime}}=\left.\mathcal{O}\left(-K_{S}\right)\right|_{B^{\prime}}=\left.\mathcal{O}(S)\right|_{B^{\prime}}=\left.\mathcal{O}_{\mathbb{P}^{3}}(2)\left(-p_{1}-\cdots-p_{8}\right)\right|_{B},
$$

which has degree 0 and is nontorsion for very general $p_{1}, \ldots, p_{8}$, and so no multiple of $B^{\prime}$ moves in $S$. It follows that the only curve on $S$ with class proportional to $-K_{S}$ is $B^{\prime}$ itself. We conclude that $C \subset S$ is a $(-2)$-curve. Since $-K_{S}$ is nef, the number of $(-2)$-curves on $S$ is finite by Proposition 4 .

The family $f$ also has four singular fibers $S_{s}$, each isomorphic to an eightpoint blow-up of a quadric cone. By genericity of the points, we may assume that the curve $B^{\prime}$ does not pass through the singular point of any of these fibers. Let $\sigma: \tilde{S}_{S} \rightarrow S_{S}$ be the blow-up at the singular point, so that $\tilde{S}_{s}$ is isomorphic to the blow-up of $\mathbb{P}(\mathcal{O} \oplus \mathcal{O}(2))$ at eight points. Then $\sigma^{*}\left(B^{\prime}\right)$ is anticanonical, and $\tilde{S}_{S}$ is a smooth rational surface with $-K_{\tilde{S}_{s}}$ nef and $K_{\tilde{S}_{s}}^{2}=0$. It follows that $-K_{\tilde{S}_{s}}$ has no movable multiple by the same argument used to prove this in the smooth fibers. So the strict transform of $C$ on $\tilde{S}_{S}$ must be a $(-2)$-curve, and, as before, there are only finitely many such curves since $-K_{\tilde{S}_{s}}$ is nef.

Since the set of $(-2)$-curves in any fiber is finite, we are reduced to showing that there are only countably many smooth fibers of $f$ containing $(-2)$-curves.

Note that $C$ corresponds to a divisor through the points $p_{1}, \ldots, p_{8}$ on some quadric surface $Q$. Restricting the section defining $C$ in $Q$ to $B$ gives a relation in $\operatorname{Pic}^{0}(B) \simeq B$ between the points $p_{1}, \ldots, p_{8}$ and line bundles coming from $Q$. However, by Lemma 9 there are only countably many fibers where this happens.

\section{Cremona Actions}

Additional curves with $L \cdot C=0$ will be constructed using repeated applications of the standard Cremona transformation on $\mathbb{P}^{3}$, yielding "elementary $(-1)$ curves", considered by Laface and Ugaglia [3]. The standard Cremona transformation $\mathrm{Cr}: \mathbb{P}^{3} \rightarrow-\rightarrow \mathbb{P}^{3}$ is given by

$$
\operatorname{Cr}\left(x_{0}, x_{1}, x_{2}, x_{3}\right)=\left(x_{0}^{-1}, x_{1}^{-1}, x_{2}^{-1}, x_{3}^{-1}\right) .
$$


Let $\pi: X \rightarrow \mathbb{P}^{3}$ be the blow-up of $\mathbb{P}^{3}$ at the four standard coordinate points. The rational map $\mathrm{Cr} \circ \pi: X \rightarrow \mathbb{P}^{3}$ can be factored as follows:

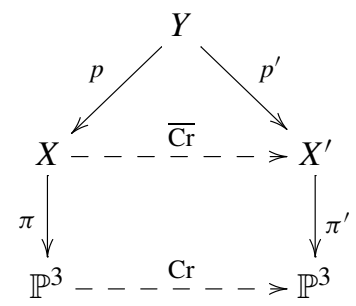

Here $p$ is the blow-up of $X$ along the transforms of the six lines through pairs of the four coordinate points. The exceptional divisors of $p$ are isomorphic to $\mathbb{P}^{1} \times \mathbb{P}^{1}$, and $p^{\prime}$ is the contraction of the "other ruling" of each $\mathbb{P}^{1} \times \mathbb{P}^{1}$. The induced map $\overline{\mathrm{Cr}}$ is a flop of these curves, and $\pi^{\prime}$ then blows down the strict transforms of the four planes through three of the four points, realizing $X^{\prime}$ as the blowup of $\mathbb{P}^{3}$ at four points as well.

The Cremona transformation has the following properties: (i) $\overline{\mathrm{Cr}}$ is an isomorphism in codimension 1, (ii) it preserves the canonical class (i.e., $\overline{\mathrm{Cr}}^{*}\left(K_{X^{\prime}}\right)=$ $K_{X}$ ), and (iii) it induces isomorphisms $M: N^{1}(X) \rightarrow N^{1}\left(X^{\prime}\right)$ and $\check{M}: N_{1}(X) \rightarrow$ $N_{1}\left(X^{\prime}\right)$, given in the standard bases by the matrices $M$ and $\tilde{M}$, where

$$
M=\left(\begin{array}{ccccc}
3 & 1 & 1 & 1 & 1 \\
-2 & 0 & -1 & -1 & -1 \\
-2 & -1 & 0 & -1 & -1 \\
-2 & -1 & -1 & 0 & -1 \\
-2 & -1 & -1 & -1 & 0
\end{array}\right), \quad \tilde{M}=\left(\begin{array}{ccccc}
3 & 2 & 2 & 2 & 2 \\
-1 & 0 & -1 & -1 & -1 \\
-1 & -1 & 0 & -1 & -1 \\
-1 & -1 & -1 & 0 & -1 \\
-1 & -1 & -1 & -1 & 0
\end{array}\right) .
$$

If $\mathbf{p}=\left(p_{1}, \ldots, p_{8}\right)$ is an eight-tuple of distinct points in $\mathbb{P}^{3}$ with the first four not coplanar, we denote by $\mathrm{Cr}_{\mathbf{p}}: \mathbb{P}^{3} \rightarrow \mathbb{P}^{3}$ the transformation $A^{-1} \circ \mathrm{Cr} \circ A$ where $A$ is the linear transformation taking $p_{1}, \ldots, p_{4}$ to the standard coordinate points. (If the points are in general position, then $A$ is uniquely determined if we additionally impose that it also fixes the point $(1,1,1,1)$.) Write $\mathbf{q}$ for the new eight-tuple $\left(p_{1}, \ldots, p_{4}, \mathrm{Cr}_{\mathbf{p}}\left(p_{5}\right), \ldots, \mathrm{Cr}_{\mathbf{p}}\left(p_{8}\right)\right)$.

Let $X_{\mathbf{p}}$ denote the blow-up of $\mathbb{P}^{3}$ at the eight points of $\mathbf{p}$, and $X_{\mathbf{q}}$ denote the blow-up of $\mathbb{P}^{3}$ at the eight points of $\mathbf{q}$. The previous discussion shows that the map $\mathrm{Cr}_{\mathbf{p}}: \mathbb{P}^{3} \rightarrow \mathbb{P}^{3}$ induces a birational map $\overline{\mathrm{Cr}}_{\mathbf{p}}: X_{\mathbf{p}} \rightarrow X_{\mathbf{q}}$, which flops the six lines between two of the four points $p_{1}, \ldots, p_{4}$.

The crucial observation is that a very general configuration of eight points in $\mathbb{P}^{3}$ has infinite orbit under the group generated by Cremona transformations. This fact was essentially known to Coble [1]; see [2] for a more modern account.

\section{Proof of Theorem 1}

We are now in position to complete the proof of Theorem 1. Again, we let $X$ denote the blow-up of $\mathbb{P}^{3}$ in a very general configuration $\mathbf{p}=\left(p_{1}, \ldots, p_{8}\right)$ of eight points. We have already seen that the set of curves $C$ such that $L \cdot C=0$ 
correspond to (-2)-curves on the fibers of $f: X \rightarrow \mathbb{P}^{1}$ and that this set is at most countably infinite. It remains only to show that this set is in fact infinite.

Lemma 11. There is an infinite set of curves $C \subset X$ with $L \cdot C=0$.

Proof. Starting from the very general configuration $\mathbf{p}_{0}=\mathbf{p}$, construct a sequence of configurations $\mathbf{p}_{0}, \mathbf{p}_{1}, \ldots, \mathbf{p}_{n}, \ldots$ so that $\mathbf{p}_{i-1}$ is obtained from $\mathbf{p}_{i}$ by making a Cremona transformation centered at the first four points of $\mathbf{p}_{i}$ and then permuting the eight-tuple to move the first entry to the end of the list. The "very general" assumption on $\mathbf{p}_{0}$ guarantees that no four points ever become coplanar (using [3, Lemma 2.6]), and so the requisite Cremona transformations are well defined.

This gives rise to a sequence of rational maps

$$
\cdots \stackrel{\mathrm{Cr}_{\mathbf{p}_{n+1}}}{\rightarrow} X_{\mathbf{p}_{n}} \stackrel{\mathrm{Cr}_{\mathbf{p}_{n}}}{\rightarrow} X_{\mathbf{p}_{n-1}} \stackrel{\mathrm{Cr}_{\mathbf{p}_{n-1}}}{\rightarrow} \cdots \stackrel{\mathrm{Cr}_{\mathbf{p}_{1}}}{\rightarrow}>X_{\mathbf{p}_{0}}=X .
$$

If $C$ is a curve on $X_{\mathbf{p}_{n}}$ such that the strict transform of $C$ on $X_{\mathbf{p}_{i}}$ is disjoint from the indeterminacy locus of $X_{\mathbf{p}_{i}}-\rightarrow X_{\mathbf{p}_{i-1}}$ for all $1 \leq i \leq n$, then the strict transform of $C$ on $X_{\mathbf{p}}$ has numerical class $\tilde{M}_{\sigma}^{n}([C])$, where

$$
\tilde{M}_{\sigma}=\left(\begin{array}{l|l}
\tilde{M} & 0 \\
\hline 0 & I_{4}
\end{array}\right)\left(\begin{array}{c|c}
1 & 0 \\
\hline 0 & \Pi_{\sigma}
\end{array}\right)=\left(\begin{array}{ccccccccc}
3 & 2 & 2 & 2 & 2 & 0 & 0 & 0 & 0 \\
-1 & -1 & 0 & -1 & -1 & 0 & 0 & 0 & 0 \\
-1 & -1 & -1 & 0 & -1 & 0 & 0 & 0 & 0 \\
-1 & -1 & -1 & -1 & 0 & 0 & 0 & 0 & 0 \\
0 & 0 & 0 & 0 & 0 & 1 & 0 & 0 & 0 \\
0 & 0 & 0 & 0 & 0 & 0 & 1 & 0 & 0 \\
0 & 0 & 0 & 0 & 0 & 0 & 0 & 1 & 0 \\
0 & 0 & 0 & 0 & 0 & 0 & 0 & 0 & 1 \\
-1 & 0 & -1 & -1 & -1 & 0 & 0 & 0 & 0
\end{array}\right)
$$

with $\Pi_{\sigma}$ the matrix encoding the permutation of the points.

If we take $\ell_{n}$ to be a line through $p_{7}$ and $p_{8}$ on $X_{\mathbf{p}_{n}}$, then its strict transform on $X_{\mathbf{p}}$ is a curve $C_{n}$ of class $\tilde{M}_{\sigma}^{n}\left(h-e_{7}-e_{8}\right)$; that the strict transforms of $\ell_{n}$ are disjoint from the indeterminacy loci is checked in [3]. It is easy to verify that the matrix $\tilde{M}_{\sigma}$ has a $3 \times 3$ Jordan block associated to the eigenvalue 1 , and a direct calculation then shows that the degrees of the classes $\left[C_{n}\right]=\tilde{M}_{\sigma}^{n}\left(h-e_{7}-e_{8}\right)$ grow without bound as $n$ is increased, so the curves $C_{n}$ are distinct.

However, for every value of $n$, we have $-K_{X_{\mathbf{p}}} \cdot C_{n}=0$ : the curve $\ell_{n} \subset X_{\mathbf{p}_{n}}$ is a rational curve with normal bundle $\mathcal{O}(-1) \oplus \mathcal{O}(-1)$, and the same is true for its strict transform $C_{n} \subset X_{\mathbf{p}}$ because $\ell_{n}$ does not meet the indeterminacy locus of the map $X_{\mathbf{p}_{n}} \rightarrow X_{\mathbf{p}}$. It follows that these give an infinite set of curves with $L \cdot C=0$.

REMARK 12. It is well known that the classes in $K_{X}^{\perp}$ on a very general pointset blow-up of projective space form a root system (in our case, it is the $T$-shaped Dynkin diagram $T_{4,4,2}$ ), and the Cremona transformations and permutations of the points induce elements in the corresponding Weyl group. Moreover, the curves on which $K_{X}$ is zero are exactly the orbit of the class of a line in $N_{1}(X)$ under this 
Weyl group. (See [2] or [6] for more precise statements.) The composition of the Cremona transformation and a permutation of the points used corresponds to the action of a Coxeter element in this group (cf. [5]).

A more detailed account can be found in [4]: the curves here are (up to permutation of the indices) the curves " $C_{n}$ " constructed in Lemma 5.2. Although [4] deals with the blow-up of $\mathbb{P}^{3}$ at nine points, the same argument works with only eight points; the only difference is that the matrix $\tilde{M}_{\sigma}$ considered here has a $3 \times 3$ Jordan block associated to the eigenvalue 1, rather than an eigenvalue greater than 1 .

Together, Lemmas 10 and 11 complete the proof of Theorem 1. The corollaries stated in the introduction follow immediately.

Proof of Corollary 2. Fix a very general smooth representative $S$ of $\left|-\frac{1}{2} K_{X}\right|$, and let $U=X-S \subset X$. It is clear that every complete curve $C$ in $U$ must satisfy $S \cdot C=-\frac{1}{2} K_{X} \cdot C=0$, and we have already shown that the set of curves with this property is countably infinite. Moreover, none of these curves $C$ except $B^{\prime}$ is contained in $S$, but they all satisfy $S \cdot C=0$. Consequently, all such $C$ are contained in $U$.

Proof of Corollary 3. Let $H$ be a very ample divisor on $X$ and consider the variety $Y=\mathbb{P}\left(\mathcal{O}_{X} \oplus \mathcal{O}_{X}(H)\right)$. The fourfold $Y$ admits two obvious maps: first, there is a $\mathbb{P}^{1}$-bundle $p: Y \rightarrow X$; second, there is a contraction $q: Y \rightarrow C X$ of the section $E \subset Y$ determined by the quotient $\mathcal{O}_{X} \oplus \mathcal{O}_{X}(H) \rightarrow \mathcal{O}_{X}$, yielding the projective cone $C X$ over $X$.

Fix an ample divisor $G$ on $C X$, and take $M=p^{*}(L)+q^{*}(G)$. The pullback $p^{*}(L)$ is certainly nef, and since $q$ is birational and $G$ is ample, $q^{*}(G)$ is big and nef. The line bundle $M$, being the sum of a nef line bundle and a big and nef one, is itself big and nef.

Suppose now that $C$ is a curve with $M \cdot C=0$. It must be that $q^{*}(G) \cdot C=0$, so $C$ is contracted by $q$ and lies in the exceptional section $E \subset Y$. Under the identification $E \cong X$, the restriction $\left.M\right|_{E}=\left.p^{*}(L)\right|_{E}$ is simply the line bundle $L$, and so the set of curves $C \subset E$ with $M \cdot C=0$ is countable.

The same construction using $\mathbb{P}\left(\mathcal{O}_{X} \oplus \mathcal{O}_{X}(H)^{\oplus r}\right)$ with $r \geq 1$ gives examples as in Corollary 3 for any dimension greater than three.

REMARK 13. If $L$ is a big and nef line bundle on a threefold $X$, then the set of curves with $L \cdot C=0$ is either finite or uncountable. Indeed, $L$ is $\mathbb{Q}$-linearly equivalent to a sum $A+E$ with $A$ ample and $E$ effective. Any curve with $(A+$ $E) \cdot C=0$ must be contained in the support of $E$. For any component $E_{i} \subset E$, the divisor $\left.L\right|_{E_{i}}$ is nef and hence zero on either finitely many or uncountably many curves; this follows from the two-dimensional statement applied on a resolution of $E_{i}$. 


\section{Remarks}

\subsection{Blow-Ups of $\mathbb{P}^{1} \times \mathbb{P}^{1} \times \mathbb{P}^{1}$}

We obtain a similar example by considering a six-point blow-up of $\mathbb{P}^{1} \times \mathbb{P}^{1} \times$ $\mathbb{P}^{1}$. Here the canonical divisor on the blow-up $Y$ is given by $2 D$ where $D=$ $\pi^{*} \mathcal{O}(1,1,1)-E_{1}-\cdots-E_{6}$. Again there is a one-dimensional family of $(1,1,1)$ divisors passing though the six points. Each $(1,1,1)$-divisor corresponds to a Del Pezzo surface of degree 6 (in fact, each map to $\mathbb{P}^{1} \times \mathbb{P}^{1}$ is the blow-up of $\mathbb{P}^{1} \times \mathbb{P}^{1}$ in two points). It follows that $Y$ is fibered into blow-ups of $\mathbb{P}^{1} \times \mathbb{P}^{1}$ in eight points, as before.

Again there are many curves on $Y$ such that $-K_{Y} \cdot C=0$. For example, when an exceptional divisor of a Del Pezzo surface passes through one of the points, the strict transform is a $(-2)$-curve on $Y$ that satisfies $K_{Y} \cdot C=0$. Here an infinite sequence of such curves can be obtained by applying the Cremona transformations of the form

$$
\left(x_{0}, x_{1}\right) \times\left(y_{0}, y_{1}\right) \times\left(z_{0}, z_{1}\right) \mapsto\left(x_{1}, x_{0}\right) \times\left(y_{0} / x_{0}, y_{1} / x_{1}\right) \times\left(z_{0} / x_{0}, z_{1} / x_{1}\right) .
$$

This transformation, and its permutations, generates an infinite representation in $G L\left(N^{1}(Y)_{\mathbb{R}}\right)$, as shown by Mukai in [6], and so arguing as before, we obtain infinitely many curves on $Y$ such that $-K_{Y} \cdot C=0$.

This threefold is not isomorphic to the previous example. There is however a birational map from $X=\mathrm{Bl}_{8} \mathbb{P}^{3}$ to $Y$ that is an isomorphism in codimension 1 . Both $\mathrm{Bl}_{4} \mathbb{P}^{3}$ and $\mathrm{Bl}_{2}\left(\mathbb{P}^{1} \times \mathbb{P}^{1} \times \mathbb{P}^{1}\right)$ have toric fans that are refinements of the fan in $\mathbb{R}^{3}$ spanned by the faces of the unit cube. From the toric description it is straightforward to see that the map $\phi: X \rightarrow Y$ flops three of the six rational curves that are flopped by the Cremona involution.
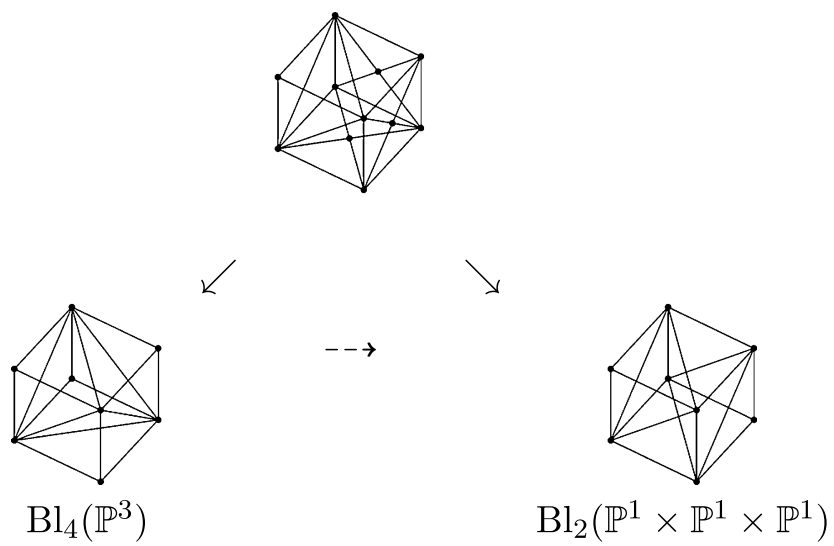

In particular, $-K_{Y}$ is also nef, and zero on exactly countably infinitely many curves, namely the strict transforms of the curves $C_{n} \subset X$. We still have $-K_{Y}$. $C_{n}=0$ since the $C_{n}$ are disjoint from the indeterminacy locus of the Cremona map, and hence from the three curves flopped by $\phi: X \rightarrow Y$. 
There is a classical correspondence between eight-tuples of points in $\mathbb{P}^{3}$ (up to automorphism) and six-tuples of points in $\mathbb{P}^{1} \times \mathbb{P}^{1} \times \mathbb{P}^{1}$, the association map. Our $X$ is the blow-up of $\mathbb{P}^{3}$ at eight very general points, and $Y$ is the blow-up of $\mathbb{P}^{1} \times \mathbb{P}^{1} \times \mathbb{P}^{1}$ at the associated set of six points.

REMARK 14. We are grateful to Vesselin Dimitrov for pointing out another affirmative example to Totaro's question. Let $\pi: X \rightarrow C$ be an nonisotrivial family of smooth abelian surfaces over a smooth projective curve $C$, and let $Z \subset X$ be the zero section. Such a family of abelian surfaces exists because the boundary of the Satake compactification of the moduli space has codimension 2.

Fix a symmetric, ample divisor $A$ on the generic fiber $X_{\eta}$. Taking the closure of $A$, we obtain a divisor $A_{0}$ on $X$. Let $B=A_{0}-\left(A_{0} \cdot Z\right) \pi^{*}(c)$ be the divisor corrected so that $B$ has intersection 0 with $Z$. Write $n: X \rightarrow X$ for the multiplication-by- $n$ map. The line bundle $B$ satisfies $n^{*} B=n^{2} B$.

We claim that $B$ is nef. First, we check that $B$ is $\pi$-ample, that is, that $\left.B\right|_{\pi^{-1}(c)}$ is ample for every $c$. This is surely the case for general $c$ since $\left.B\right|_{\pi^{-1}(c)}=$ $\left.A\right|_{\pi^{-1}(c)}$. If a divisor is ample on a single fiber of a smooth family of abelian varieties, it is ample on every fiber.

Hence, $B$ is $\pi$-ample. We must also show that $B \cdot Y \geq 0$ if $Y$ is a horizontal curve. In that case, the intersection $B \cdot Y=\left(1 / \operatorname{deg}\left(\left.\pi\right|_{Y}\right)\right) h(Y)$, where $h(Y)$ is the canonical height of $Y$ with respect to $A$ [9, (2.5.3)]. This height is 0 if and only if $Y$ is a torsion section of $X$. In particular, $B$ has intersection 0 with any of the countably many torsion sections of $\pi$.

In this case, the divisor $B$ is not effective and so does not yield an example as in Corollary 2.

REMARK 15. If $\phi: X \rightarrow X^{+}$is the flop of a disjoint set of rational curves $B_{i}$ with normal bundles $\mathcal{O}_{\mathbb{P}^{1}}(-1) \oplus \mathcal{O}_{\mathbb{P}^{1}}(-1)$, and $H$ is a divisor on $X$ with strict transform $\tilde{H}$ on $X^{+}$, then $\tilde{H}^{3}=H^{3}-\sum_{i}\left(H \cdot C_{i}\right)^{3}$. For every $n$, the map $X_{\mathbf{p}_{n}} \rightarrow X$ flops such a collection of disjoint rational curves. If $H_{n}$ is a plane in $X_{\mathbf{p}_{n}}$, it has positive intersection with each of these $6 n$ curves. It follows that the strict transforms $G_{n} \subset X$ of the divisors $H_{n} \subset X_{\mathbf{p}_{n}}$ define a sequence of irreducible divisors on $X$ for which the top self-intersection is not bounded below. This shows that the most naïve three-dimensional analog of the Bounded Negativity Conjecture for surfaces does not hold: on a threefold, there is no lower bound on $D^{3}$ for irreducible divisors $D$.

\subsection{A Question}

The example here shows that it is possible for a linear subspace of $N_{1}(X)$ to contain precisely a countable number of irreducible curves: $-K_{X}^{\perp} \subset N_{1}(X)$ is such a subspace. Since $-K_{X}$ is nef, $-K_{X}^{\perp} \cap \overline{\mathrm{NE}}(X)$ is in fact an extremal face of the cone of curves $\overline{\mathrm{NE}}(X)$ containing a countable number of irreducible curves. Related is the following: 
Question. Let $X$ be a smooth projective variety, and let $\alpha \in N_{1}(X)$ be a numerical cycle class. Can it happen that the set of irreducible curves on $X$ with class proportional to $\alpha$ is countably infinite?

Again, this cannot happen on a surface.

Acknowledgments. We would like to thank I. Dolgachev, B. Lehmann, V. Dimitrov, and S. Cantat for useful comments. The first author was supported in part by the NSF under grant No. DMS-1128155.

\section{References}

[1] A. B. Coble, Algebraic geometry and theta functions, Amer. Math. Soc. Colloq. Publ., 10, American Mathematical Society, Providence, R.I., 1982, Reprint of the 1929 edition.

[2] I. Dolgachev and D. Ortland, Point sets in projective spaces and theta functions, Astérisque 165 (1988), 210 pp., (1989).

[3] A. Laface and L. Ugaglia, Elementary (-1)-curves of $\mathbb{P}^{3}$, Comm. Algebra 35 (2007), no. $1,313-324$.

[4] J. Lesieutre, The diminished base locus is not always closed, Compos. Math. 150 (2014), no. 10, 1729-1741.

[5] C. T. McMullen, Dynamics on blowups of the projective plane, Publ. Math. Inst. Hautes Études Sci. 105 (2007), 49-89.

[6] S. Mukai, Counterexample to Hilbert's fourteenth problem for the 3-dimensional additive group, RIMS Kyoto preprint 1343, 2001.

[7] B. Totaro, Moving codimension-one subvarieties over finite fields, Amer. J. Math. 131 (2009), 1815-1833.

[8] _ The topology of smooth divisors and the arithmetic of abelian varieties, Michigan Math. J. 48 (2000), 611-624.

[9] K. Yamaki, Trace of abelian varieties over function fields and the geometric Bogomolov conjecture, 2014, arXiv:1405.0896.

\section{J. Lesieutre}

Institute for Advanced Study

Einstein Drive

Princeton, New Jersey 08540

USA

johnl@math.ias.edu

\section{Current address}

Department of Mathematics, Statistics, and Computer Science

University of Illinois at Chicago

Room 411 SEO M/C 249

851 S. Morgan St.

Chicago, Illinois 60607-7045

USA
J. C. Ottem

Department of Mathematics

University of Oslo

Box 1053, Blindern, 0316 Oslo

Norway

johnco@math.uio.no

\section{jdl@uic.edu}

